\title{
Brahma-related gene 1 inhibits proliferation and migration of human aortic smooth muscle cells by directly up-regulating Ras-related associated with diabetes in the pathophysiologic processes of aortic dissection
}

Wei-Lin Liao, MD, Meng-Wei Tan, MD, Yang Yuan, MD, Guo-Kun Wang, MD, Chong Wang, MD, Hao Tang, MD, and Zhi-Yun Xu, MD

\begin{abstract}
Objective: To elucidate the mechanisms of Brahma-related gene 1 (Brg1) involvement in the pathophysiologic processes of aortic dissection.

Methods: Seventeen dissecting, 4 dilated, and 10 healthy human aorta samples were collected. Expression of Brg1 in the medium of aorta was evaluated by quantitative real-time polymerase chain reaction, Western blot, and immunohistochemical staining, respectively. The regulation effect of Brg1 on proliferation and migration of human aortic smooth muscle cells (HASMCs) was analyzed in 3 ways: using cell counting, a migration chamber, and a wound scratch assay. A polymerase chain reaction array was used for screening potential target genes of Brg1. A chromatin immunoprecipitation assay was adopted for direct deoxyribonucleic acid-protein binding detection.
\end{abstract}

Results: Expression levels of Brg1 were increased in aortic dissection and aortic dilation patients. In vitro results indicated that overexpression of Brg1 inhibited proliferation and migration of HASMCs. The candidate proliferation- and migration-related Brg1 target gene found was Ras-related associated with diabetes (RRAD), expression levels of which were enhanced in dissecting aortic specimens. The direct regulation effect of Brg1 on RRAD was verified by chromatin immunoprecipitation assay results. Furthermore, down-regulating RRAD significantly alleviated the suppression effects of Brg1 on proliferation and migration of HASMCs.

Conclusions: Our study illustrated that Brg1 inhibited the proliferation and migration capacity of HASMCs, via the mechanism of direct up-regulation of RRAD, thus playing an important role in the pathophysiologic processes of aortic dissection. (J Thorac Cardiovasc Surg 2015;150:1292-301)

丹 Supplemental material is available online.

Aortic dissection $(\mathrm{AD})$ is a life-threatening disease with an estimated incidence of approximately 3 to 4 cases per 100,000 people each year. $^{1,2}$ Despite substantial

From the Department of Cardiothoracic Surgery, Changhai Hospital, Second Military Medical University, Shanghai, People's Republic of China.

This work was supported by the National Natural Science Foundation of China (grant 81300233 to Dr Tan).

Wei-Lin Liao and Meng-Wei Tan contributed equally to this work.

Received for publication April 29, 2015; revisions received July 29, 2015; accepted for publication Aug 1, 2015; available ahead of print Sept 3, 2015.

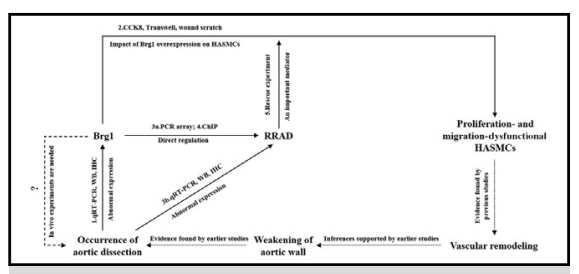

Expression of Brg1 was significantly increased in the medium of aorta of patients with AD. Overexpression of Brg1 would inhibit proliferation and migration of HASMCs in vitro through direct up-regulation of RRAD, expression of which was enhanced as well in this aortic medium.

\section{Central Message}

$\overline{\text { Brg1 inhibition of HAMSC proliferation and }}$ migration via direct up-regulation of RRAD plays an important role in the pathogenesis of $\mathrm{AD}$

\section{Perspective}

Despite substantial recent improvement in diagnosis and treatment of aortic dissection, associated mortality remains high. Given the potential for ameliorating clinical treatment of aortic dissection, further clarification of its pathogenesis is needed, as provided in this study.

See Editorial Commentary page 1301.

\footnotetext{
Address for reprints: Hao Tang, MD, or Zhi-Yun Xu, MD, Department of Cardiothoracic Surgery, Changhai Hospital, Second Military Medical University, 168 Changhai Rd, Shanghai 200433, People's Republic of China (E-mail: dr_tanghao@vip.163.com or zhiyunx@hotmail.com). $0022-5223 / \$ 36.00$ Copyright () 2015 by The American Association for Thoracic Surgery http://dx.doi.org/10.1016/j.jtcvs.2015.08.010
} 


$\begin{aligned} & \text { Abbreviations and Acronyms } \\ & \text { AD }=\text { aortic dissection } \\ & \text { Brg1 }=\text { Brahma-related gene } 1 \\ & \text { GFP }=\text { green fluorescent protein } \\ & \text { HASMC }=\text { human aortic smooth muscle cell } \\ & \text { PCR }=\text { polymerase chain reaction } \\ & \text { RNA }=\text { ribonucleic acid } \\ & \text { RRAD }=\text { Ras-related associated with diabetes } \\ & \text { shBrg1 }=\text { Brg1-specific short hairpin RNA } \\ & \text { adenovirus vector } \\ & \text { shRRAD }= \text { RRAD-specific short hairpin RNA } \\ & \text { adenovirus vector } \\ & \text { shScramble = } \text { negative control short hairpin RNA } \\ & \text { adenovirus vector } \\ & \text { SWI/SNF }= \text { switch/sucrose nonfermenting } \\ & \text { VSMC }=\text { vascular smooth muscle cell }\end{aligned}$

Vascular smooth muscle cells (VSMCs) are the major components of aortic media, and they have been previously reported to be involved in various aortic diseases. ${ }^{4}$ According to several studies, anomalous hemodynamic and hormonal status can, under some conditions, result in VSMC dysfunction, reducing their ability to proliferate and migrate. ${ }^{4-6}$ Via mediation of vascular remodeling, biological changes in VSMCs may ultimately lead to aortic wall weakening, ${ }^{7}$ generally considered to be the pathologic basis of aortic dissection. However, the specific molecular mechanisms of regulation of VSMC proliferation and migration capabilities in these pathologic conditions are not well understood.

Brahma-related gene $1(\mathrm{Brg} 1)$, the core subunit of the SWI/SNF complex (mating types switching and sucrose nonfermenting complex), contributes to epigenetic regulation by utilizing the energy of adenosine triphosphate hydrolysis to remodel chromatin and regulate transcription of target genes. ${ }^{8}$ Previous studies have illustrated the critical role of Brg1 in cardiovascular embryonic development and disease to regulate cell proliferation and differentiation. ${ }^{9-12}$ Besides, our recent studies have shown that $\operatorname{Brg} 1$ is involved in aortic aneurysms and dissection, at least through the point of inducing apoptosis of VSMCs. ${ }^{13,14}$ However, the more-precise mechanisms of Brg1 function in aortic dissection are not well understood.

In the present study, Brg1 was found to be responsible for dysfunction of VSMCs in proliferation and migration in aortic dissection via direct up-regulation of the expression of Ras-related associated with diabetes (RRAD). This small GTPase was first identified because of its association with insulin resistance in type II diabetes mellitus. ${ }^{15}$

\section{METHODS \\ Ethics Statement}

All the study protocols, including that for acquisition of informed consent, were approved by the Institutional Review Boards of Changhai Hospital affiliated with the Second Military Medical University (Shanghai, People's Republic of China). For examinations utilizing human aortic tissue specimens, all patients and organ donors' direct relatives gave written informed consent.

\section{Patients and Samples}

A total of 17 patients undergoing surgical treatment for acute Stanford type A aortic dissection at the Department of Cardiothoracic Surgery, Changhai Hospital, from March to May, 2014 were included in the study group. A segment of full-thickness, dissecting and nondissecting, but nonaneurysmal, dilated, proximal, ascending aortic tissue samples were harvested fresh during surgery from each subject. In the aortic dilation group, 4 dilated, ascending aortic specimens with full-thickness, from 4 patients who had aortic dilation, but were not bicuspid, did not have aneurysm, and did not have dissection, were obtained from the Department of Pathology, Changhai Hospital.

In the control group, 10 specimens of healthy, full-thickness, proximal, ascending thoracic aorta segments were obtained from 10 organ donors. In the 3 groups, subjects with connective tissue diseases (eg, Marfan syndrome), bicuspid aortic valve malformation, syphilis, family history of aortic diseases, and a history of cardiovascular surgery, were excluded. Demographic data of subjects in the 3 groups were collected and are shown in Tables 1, 2, and E1, respectively. For Western blot and quantitative real-time polymerase chain reaction analysis, the aortic specimens were carefully cleaned of intima and adventitia. For immunohistochemical staining, full-thickness aortic tissues were used.

\section{Cell Culture}

Human aortic smooth muscle cells (HASMCs) purchased from Cascade Biologics (Portland, Ore) were used for experiments at passages 3 through 8. The cells were grown in Medium 231 with smooth muscle growth supplements (Cascade Biologics).

\section{Overexpression of Brg1 and Ribonucleic Acid Interference}

An adenovirus vector coexpressing $\mathrm{Brg} 1$ as well as green fluorescent protein (GFP; Ad-Brg1), and a control vector expressing GFP (Ad-GFP) were constructed, using the AdMax system (Sunbio Medical Biotechnology Co, Ltd, Shanghai, People's Republic of China). The Brg1-specific short hairpin RNA (ribonucleic acid) adenovirus vector ( $\operatorname{shBrg} 1$ ), the RRAD-specific short hairpin RNA adenovirus vector (shRRAD), and the negative control short hairpin RNA adenovirus vector (shScramble) were purchased from Sunbio Medical Biotechnology Co, Ltd.

\section{Immunohistochemical Staining}

The immunohistochemical staining was performed as previously described. ${ }^{16}$ The primary antibodies used were Brg1 (Sc-17796; Santa Cruz Biotechnology, Santa Cruz, Calif) and RRAD (ab75100; Abcam, Inc, Cambridge, Mass).

\section{Quantitative Real-Time Polymerase Chain Reaction}

Total RNA extraction and complementary deoxyribonucleic acid (cDNA) synthesis were performed as previously described. ${ }^{17}$ Quantitative real-time polymerase chain reaction was performed on a LightCycler 480 Real-Time PCR System (F. Hoffmann-La Roche AG, Basel Switzerland). The sequences of primers for each gene are shown in Appendix E1. 
TABLE 1. Profiles of patients with aortic dissections

\begin{tabular}{lccccc}
\hline $\begin{array}{c}\text { Age (y) } \\
\text { and } \\
\text { gender }\end{array}$ & Hypertension & $\begin{array}{c}\text { TC } \\
(\mathbf{m m o l} / \mathbf{L})\end{array}$ & $\begin{array}{c}\text { TG } \\
(\mathbf{m m o l} / \mathbf{L})\end{array}$ & $\begin{array}{c}\text { Diabetes } \\
\text { symptoms } \\
\text { (h) }\end{array}$ \\
\hline 61, Male & Yes & 2.96 & 0.54 & No & 3 \\
54, Male & Yes & 3.19 & 3.22 & Yes & 19 \\
44, Female & No & 3.21 & 1.03 & No & 24 \\
66, Female & No & 4.13 & 1.54 & No & 15 \\
51, Male & No & 5.28 & 1.62 & No & 24 \\
59, Female & Yes & 5.11 & 0.89 & No & 11 \\
51, Male & Yes & 4.82 & 1.09 & Yes & 9 \\
59, Female & No & 3.47 & 0.86 & No & 7 \\
50, Male & Yes & 5.55 & 0.75 & No & 24 \\
39, Male & Yes & 4.52 & 1.67 & No & 9 \\
43, Male & Yes & 4.21 & 1.09 & No & 8 \\
38, Male & Yes & 3.12 & 0.56 & No & 18 \\
52, Male & Yes & 3.67 & 1.62 & No & 26 \\
59, Male & No & 5.24 & 1.65 & No & 6 \\
61, Female & Yes & 5.91 & 1.93 & No & 12 \\
47, Male & Yes & 4.38 & 0.96 & No & 13 \\
57, Male & Yes & 4.57 & 1.63 & Yes & 17
\end{tabular}

All patients had a Stanford classification of A. $T C$, Serum total cholesterol; $T G$, serum triglyceride.

\section{Western Blot}

Western blot was performed as described elsewhere. ${ }^{13}$ The following primary antibodies were used: Brg1 (Sc-17796), RRAD (ab75100), $\beta$-actin (60008-1-Ig; Proteintech Group, Chicago, Ill), and Lamin B (ab151735; Abcam, Inc).

\section{Chromatin Immunoprecipitation}

The chromatin immunoprecipitation assay was performed as described elsewhere, ${ }^{16}$ with modifications (Appendix E1).

\section{Polymerase Chain Reaction Array}

The cells used for PCR array analysis were (1) Ad-GFP HASMCs, as control cells: and (2) Ad-Brg1 HASMCs, as study cells. Total cellular

TABLE 2. Donor profiles

\begin{tabular}{|c|c|c|c|c|c|}
\hline $\begin{array}{l}\text { Age }(y) \\
\text { and } \\
\text { gender }\end{array}$ & $\begin{array}{l}\text { Hyper- } \\
\text { tension }\end{array}$ & $\begin{array}{c}\mathrm{TC} \\
(\mathrm{mmol} / \mathrm{L}) \\
\end{array}$ & $\begin{array}{c}\text { TG } \\
(\mathrm{mmol} / \mathrm{L})\end{array}$ & Diabetes & $\begin{array}{c}\text { Cause of } \\
\text { death }\end{array}$ \\
\hline 45, Female & No & 4.21 & 1.63 & No & Car accident \\
\hline 59, Male & No & 3.63 & 0.78 & Yes & Glioma \\
\hline 39, Male & Yes & 3.69 & 1.36 & No & Car accident \\
\hline 52, Female & No & 4.32 & 1.61 & No & $\begin{array}{l}\text { ARDS caused } \\
\text { by drowning }\end{array}$ \\
\hline 25, Male & Yes & 3.52 & 0.98 & No & Car accident \\
\hline 43, Female & Yes & 3.79 & 1.84 & Yes & Car accident \\
\hline 40, Female & No & 4.25 & 0.77 & No & $\begin{array}{l}\text { Cervical spinal } \\
\text { fracture }\end{array}$ \\
\hline 33, Male & No & 4.99 & 1.07 & No & Car accident \\
\hline 41, Male & No & 4.27 & 1.00 & No & $\begin{array}{l}\text { ARDS caused } \\
\text { by inhalation } \\
\text { of chlorine }\end{array}$ \\
\hline 49 , Female & No & 3.36 & 1.10 & No & Glioma \\
\hline
\end{tabular}

$T C$, Serum total cholesterol; $T G$, serum triglyceride; $A R D S$, acute respiratory distress syndrome.
RNA was isolated using the TRIzol reagent (Invitrogen, Carlsbad, Calif) followed by purification using the RNeasy Mini Kit (QIAGEN, Munich Germany), according to manufacturer instructions. RNA was converted to complementary deoxyribonucleic acid using the $\mathrm{RT}^{2}$ First Strand Kit (QIAGEN). Real-time PCR running on a LightCycler 480 Real-Time PCR System was performed using a 96-well RT ${ }^{2}$ Profiler PCR array (QIAGEN) to clarify the expression profiles of 84 candidate genes filtered from previously published literature, which were related to cell proliferation and migration. Five housekeeping genes (18s rRNA, GAPDH, $\beta$-actin, TBP, and RPLP1) were used for normalizing the expression levels of the target genes aforementioned. A threshold value of 2.0 was adopted to identify genes of interest. A heat map was generated using freeware TM4-MEV and R software (The R Project for Statistical Computing; www.r-project. $\operatorname{org} /)$.

\section{Cell Proliferation}

A cell proliferation assay was performed using the Cell Counting Kit-8 (CCK8, Beyotime, Haimen, Shanghai, China). Cells were seeded in 96-well plates at a density of 4000 cells per well in $100 \mu \mathrm{L}$ of Dulbecco's Modified Eagle's Medium. Sixteen hours later, cells were transfected with adenovirus. At $0,24,48$, and 72 hours after transfection, $10 \mu \mathrm{L}$ of CCK8 solution was added to each well, and the cultures were incubated at $37^{\circ} \mathrm{C}$. One hour later, absorbance at $450 \mathrm{~nm}$ was measured.

\section{Cell Migration}

Cell migration assay was performed using 2 approaches: (1) using a Transwell migration chamber (ThermoFisher Scientific, Waltham, Mass); and (2) using a wound scratch assay. In brief, the first was performed in a 24-well Transwell chamber (pore size: $8 \mu \mathrm{m}$ ). Twenty-four hours after transfection, $1.0 \times 10^{5}$ cells were plated into the upper chamber with a noncoated membrane. Next, the cells were incubated for 12 hours. Cells that did not migrate through the pores were removed with a cotton swab. Cells that migrated were fixed, stained in a $0.1 \%$ crystal violet solution, and counted.

In the wound scratch assay, a straight-line scratch was made on a monolayer of cells using a sterile plastic pipette tip 24 hours after transfection. The cells were washed using Dulbecco's Modified Eagle's Medium. For each well, pictures were taken at 2 time points: 0 and 48 hours after scratch. The migration distance was calculated as the difference of the gap width at the 2 time points.

\section{Statistical Analysis}

Data are presented as mean $\pm \mathrm{SD}$. Statistical comparisons were analyzed with SAS software, version 9.13 (SAS Institute, Cary, NC).

\section{RESULTS}

\section{Expression of Brg1 Is Increased in the Medium of Dissecting Aorta}

Quantitative RT-PCR and Western blot analysis were used to examine the expression levels of Brg1 in the media of healthy aorta in healthy individuals as well as in dissecting aortic wall in AD patients. The qRT-PCR results showed that Brg1 messenger RNA (mRNA) levels were significantly higher (approximately 4-fold) in AD patients, compared with those of healthy individuals (Figure 1, A). Similarly, Western blot results showed higher expression levels of $\mathrm{Brg} 1$ protein in $\mathrm{AD}$ patients (Figure 1, B). Morphologic study of immunohistochemistry showed that $\mathrm{Brg} 1$ levels were greater in the media of both dissected and nondissected portions of the aorta in $\mathrm{AD}$ patients, 
compared with healthy aorta. In addition, expression of Brg1 was enhanced in the media of dilated aorta, compared with healthy aorta (Figure 1, C).

\section{Proliferation and Migration Capability of HASMCs Is Downregulated by Brg1}

Given that the local expression of Brg1 increased considerably in the aortic media of AD patients, compared with healthy individuals, and in combination with the definite recognition of the important role of Brg1 in regulating the proliferation and migration of some critical cells in a series of diseases, we investigated the effect of Brg1 on the HASMC properties of proliferation and migration.

We transfected HASMCs with Ad-Brg1, and the transfection efficiency reached $\leq 90 \%$ (Figure $1, F$ ). The HASMCs transfected with Ad-GFP served as controls. The expression levels of Brg 1 were examined 36 hours after transfection. The relative mRNA level of Brg1 in the Ad-Brg1 HASMCs was 8.67, compared with control cells (Figure 1, D). In addition, an enhanced expression of Brg1 protein in the Ad-Brg1 cells was observed, according to Western blot results (Figure 1,E).

The cell proliferation property examination was performed using a CCK8 assay. We found that Ad-Brg1
HASMCs manifested drastically decreased proliferation levels at 24, 48, and 72 hours after transfection (Figure 2, $A)$. With regard to HASMC migration-capability evaluation, wound scratch and migration chamber assays were both applied to assess the impact of Brg1 on this cell property in 2-dimensional and 3-dimensional space, respectively. Experimental results showed that Ad-Brg1 HASMCs presented significantly decreased migration capability in both 2-dimensional (Figure 2, $B$ and $D$ ) and 3-dimensional space (Figure 3, $C$ and $E$ ), compared with Ad-GFP cells. These results indicated that Brg1 played a critical negative regulation role in the proliferation and migration capability of HASMCs.

\section{Signature of Proliferation- and Migration-Related Genes in Ad-Brg1 HASMCs and RRAD Expression in Dissecting Aorta}

To elucidate the mechanisms of the negative regulation effect of Brg1 on proliferation and migration of HASMCs, PCR array analysis was used to preliminarily determine the potential target genes of Brg1. This step was accomplished by comparing the relative expression-level differences of a total of 84 candidate genes, related to cell proliferation and migration, for Ad-Brg1 HASMCs versus the control group
A
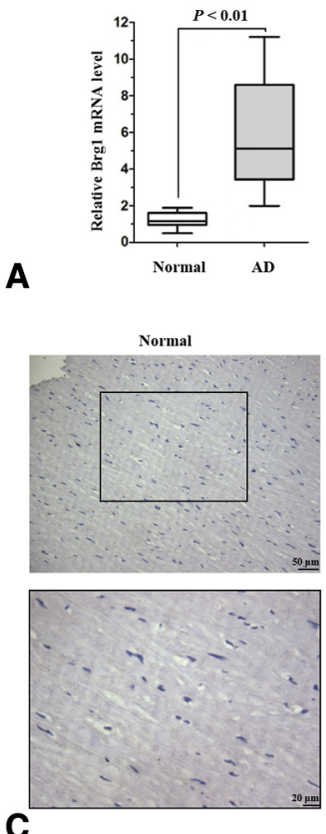

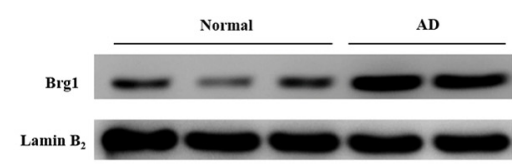

B
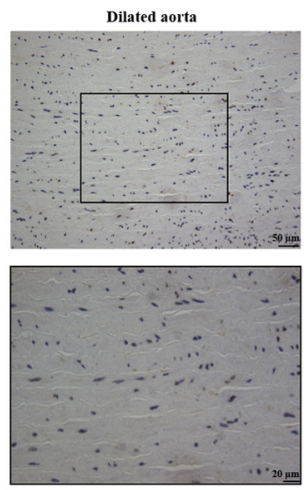

AD (non-dissected portion)
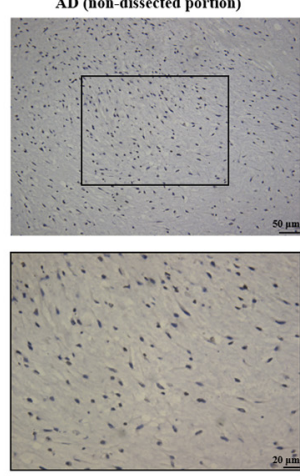

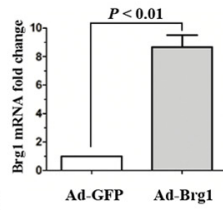

AD (dissected portion)
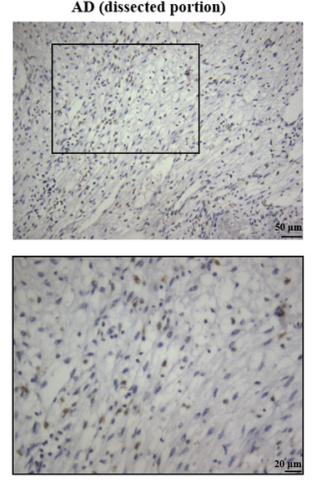

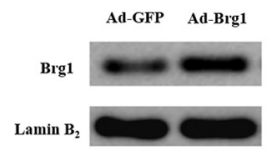

E
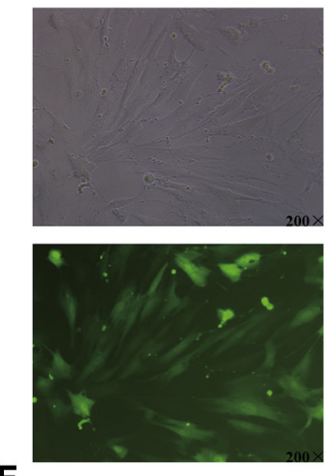

$\mathbf{F}$

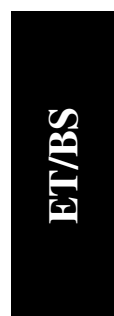

FIGURE 1. Expression levels of Brglin the media of dissecting versus healthy human aorta, and verification of Brg1 overexpression in Ad-Brg1 HASMCs. A, Analysis using qRT-PCR showed that Brg1 mRNA levels were significantly higher in AD patients $(\mathrm{n}=17)$ than in healthy individuals ( $\mathrm{n}=10)$. B, Local expressions of Brg1 protein were also upregulated in AD. C, Immunohistochemical staining results showed that Brg1 expression levels were enhanced in the nucleus of smooth muscle cells in the media of aorta of patients with either AD or aorta dilation, compared with healthy aorta. The images of nondissected and dissected aorta portions were taken from the same patient who had AD. D and E, Overexpression of Brg1 in Ad-Brg1 HASMCs was verified through qRT-PCR and Western blot, respectively ( $\mathrm{n}=3$ for each cell group); Ad-GFP served as a control. F, The transfection efficiency of adenovirus vectors in HASMCs, which was assessed under a fluorescence microscope $(\times 200)$, reached $\leq 90 \%$. Brg 1 , Brahma-related gene $1 ; m R N A$, messenger ribonucleic acid; $A D$, aortic dissection; $A d-G F P$, a control vector expressing green fluorescent protein; $A d-B r g 1$, adenovirus vector coexpressing Brg1 as well as green fluorescent protein. 


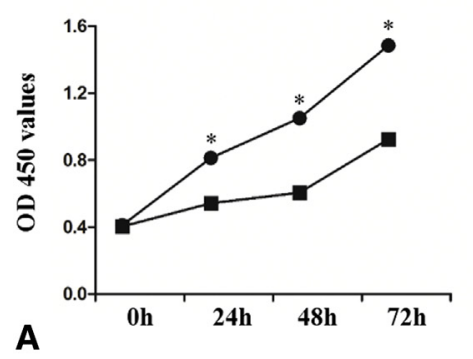

oh
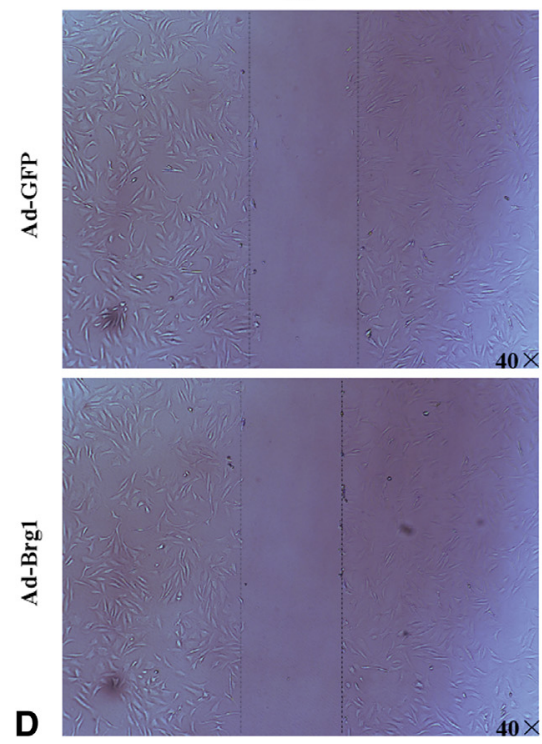

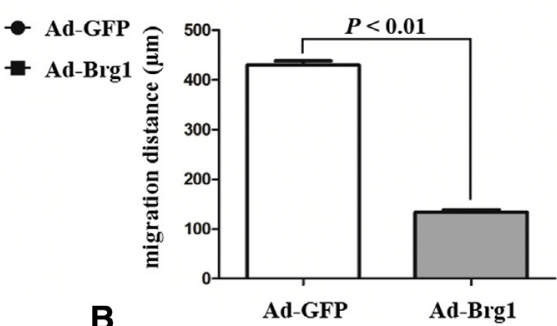

$48 \mathrm{~h}$
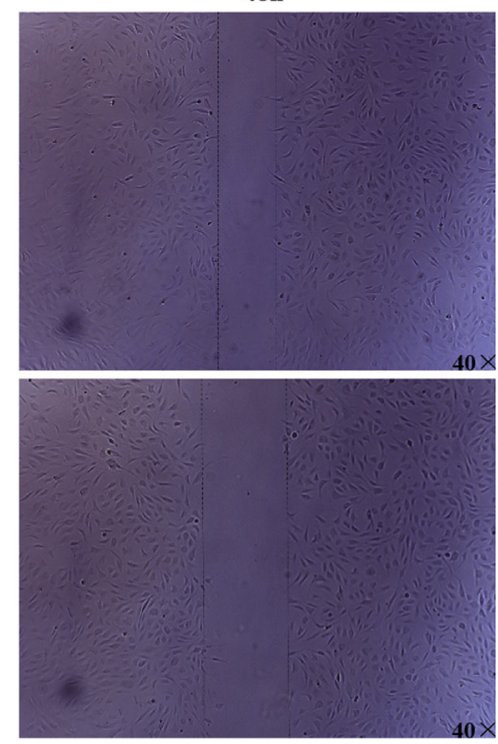
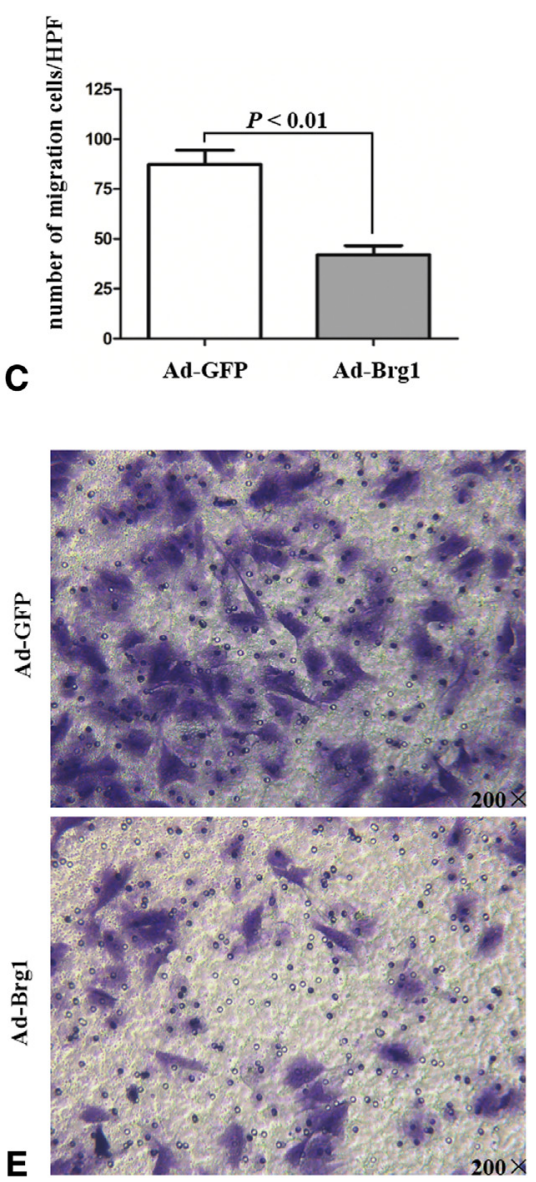

FIGURE 2. Brg1 negatively regulated proliferation and migration of HASMCs. A, A lower optical density at $450 \mathrm{~nm}$ in the CCK8 assay was observed in Ad-Brg1 HASMCs at 24, 48, and 72 hours, respectively, after transfection ( $\mathrm{n}=3$ for each cell group). B and D, According to the wound scratch assay ( $\times 40)$, Ad-Brg1 HASMCs demonstrated significantly lower migration capability in 2-dimensional space. Migration distance ( $\mu$ m) of HASMCs was quantified ( $n=3$ for each cell group). C and E, Results of the migration chamber assay showed that migration capability in 3-dimensional space $(\times 200)$ was also significantly lower in the Ad-Brg1 HASMCs. Quantified data were presented as the number of migration cells per HPF. For each cell group, $\mathrm{n}=3$. All experiments were carried out in triplicate. OD, Optical density; $A d$ - $G F P$, a control vector expressing green fluorescent protein; $A d-B r g 1$, adenovirus vector coexpressing $\operatorname{Brg} 1$ as well as green fluorescent protein; $H P F$, high-power field. ${ }^{*} P<.01$ versus control.

(Figure 3, A). Results showed that significant upregulation of mRNA expression levels in the Ad-Brg1 group, compared with the control group, for the following (fold increase): MAX (3.29); Myc (2.37); p53 (2.26); PARP6 (3.78), RRAD (4.09), and Smad3 (3.35). The mRNA expression levels of CBX8 (35.3\%), HMGB1 (24.6\%), and LIN28B (38.9\%), on the other hand, were significantly downregulated in Ad-Brg1 HASMCs compared with AdGFP cells (Figure 3, $B$ and $C$ ).

Ras-related associated with diabetes is a member of the Ras GTPase superfamily. It was first identified for its association with insulin resistance in type II diabetes mellitus and was reported to be highly expressed in heart and lung. ${ }^{15}$ Accumulating evidence has suggested that RRAD is a critical mediator, participating in cell migration and many human diseases. ${ }^{18,19}$ However, whether RRAD is involved in the pathogenesis of $\mathrm{AD}$ is unclear. To clarify this issue, we examined the expression levels of RRAD in the $\mathrm{AD}$ and normal aortic specimens.

The RRAD mRNA levels were significantly higher in the AD group (by approximately 3.6 fold), compared with healthy controls (Figure 3, D). The Western blot results further confirmed an increased RRAD protein level in the dissecting aortic wall, compared with healthy aorta (Figure 3, E). Moreover, immunohistochemical results showed increased expression of RRAD in the media of both dissected and nondissected portions of the aorta in $\mathrm{AD}$ patients. In addition, the expression of RRAD was enhanced in the media of dilated aorta, compared with normal aorta (Figure 3,F). Taken together, these results suggested that RRAD might be involved in the pathogenesis of $\mathrm{AD}$ and the process of HASMC proliferation and migration regulation, which was acknowledged as a crucial issue in $\mathrm{AD}$ pathogenesis. 


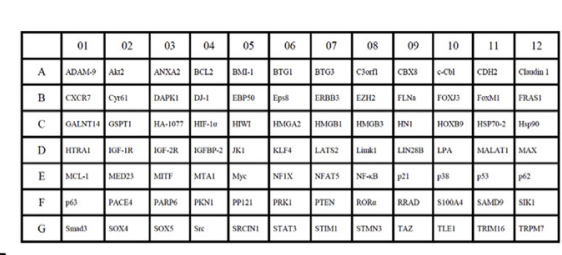

A

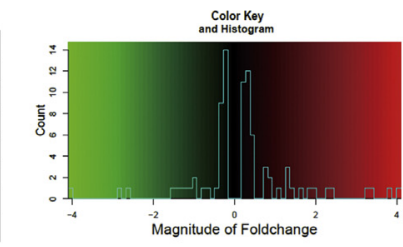

\section{C}
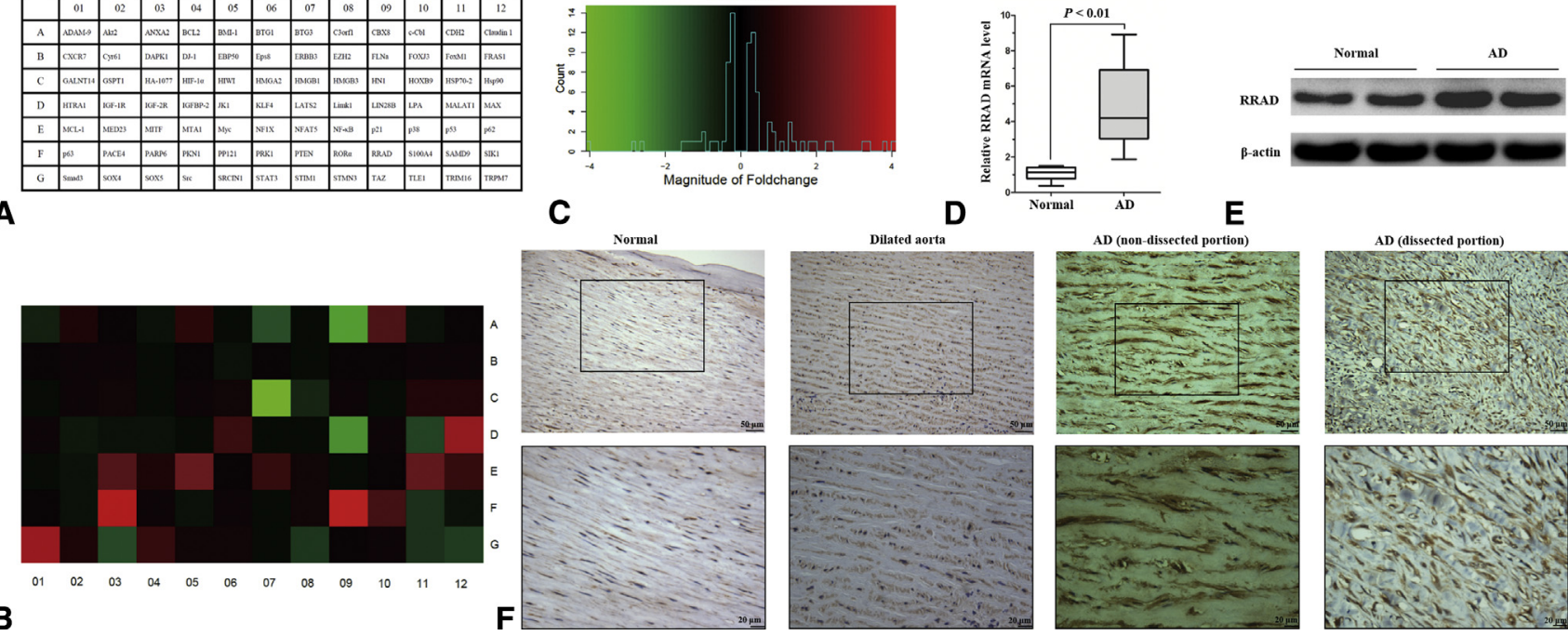

E
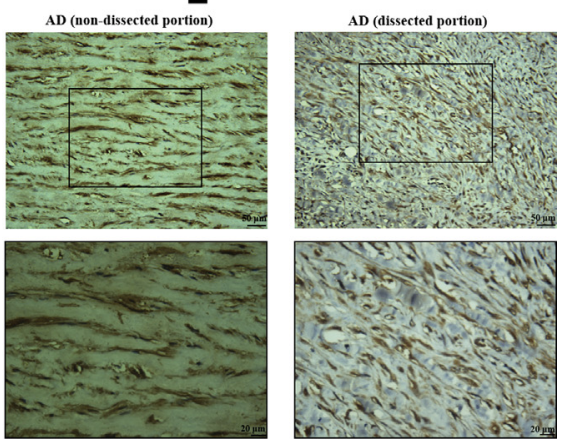

FIGURE 3. Polymerase chain reaction array analysis of proliferation- and migration-related genes in Ad-Brg1 HASMCs and enhanced RRAD expression in the medium of dissecting aorta. A, Names of the 84 proliferation- and migration-related genes. B and C, Heat map of the variations in the expression of the 84 genes between Ad-Brg1 and Ad-GFP HASMCs. D and E, mRNA and protein expression levels of RRAD were both higher in AD patients $(\mathrm{n}=17)$ than in healthy individuals $(n=10)$. F, The expression of RRAD was enhanced in the cytoplasm of smooth muscle cells in the media of aorta of patients with AD or dilated aorta, compared with healthy aorta. The images of a nondissected portion and a dissected portion of aorta were taken from the same patient who had AD. RRAD, Ras-related associated with diabetes; $m R N A$, messenger ribonucleic acid; $A D$, aortic dissection.

\section{Brg1 Directly Regulates RRAD Expression in HASMCs}

The core subunit of SWI/SNF chromatin remodeling complex, Brg1, mostly exerts its functions via selectively regulating transcription of target genes with the energy of adenosine triphosphate hydrolysis. ${ }^{8}$ However, whether Brg1 regulates the expression of RRAD in HASMCs is still uncertain, although we found a positive PCR array analysis result, as mentioned. Thus, we further verified, through qRT-PCR and Western blot that, as we speculated, RRAD mRNA and protein levels were both significantly upregulated in Ad-Brg1 HASMCs, compared with control cells (Figure 4, $A$ and $B$ ).

The fact that RRAD is regulated by Brg1 was further confirmed by experiments of knocking down Brg1 in HASMCs, by transfecting HASMCs with shBrg1. HASMCs that were transfected with shScramble were used as a negative control. Compared with shScramble HASMCs, the expression levels of Brg1 and RRAD mRNA in shBrg1 cells decreased to $27.3 \%$ and $47.7 \%$, respectively (Figure 4, $C$ and $E$ ). Additionally, Western blot results showed lower expression levels of $\operatorname{Brg} 1$ and RRAD in shBrg1 HASMCs (Figure 4, $D$ and $F$ ).

Taking into account the chromatin remodeling effect of $\mathrm{Brg} 1$, these intriguing results prompted us to consider whether RRAD was directly regulated by Brg 1 in HASMCs. To verify this hypothesis, we further used the chromatin immunoprecipitation assay to examine whether $\mathrm{Brg} 1$ is recruited to the promoter of RRAD. Using primer sets flanking a region of about 1500 bas pairs, DNA enrichment was measured by RT-PCR and semi-qPCR. An enrichment of Brgloccurred in a region of approximately -350 to -750 base pairs upstream of the transcription start site of RRAD (Figure 4, $G$ ). These findings, for the first time, demonstrated that RRAD was a direct regulation target of Brg1 during transcription in HASMCs.

\section{Repression Effects of Brg1 on Proliferation and Migration in HASMCs are Mediated by RRAD}

Based on the results given, and the fact that RRAD was previously reported to be involved in tumor cell migration, we further assessed whether the suppression effects of Brg1 on proliferation and migration in HASMCs were mediated via RRAD. To make this supposition clear, a rescue experiment was performed via transfection shRRAD in Ad-Brg1 HASMCs. As expected, the RRAD mRNA and protein levels in Ad-Brg1/shRRAD HASMCs were effectively silenced compared with Ad-Brg1/shScramble cells (Figure 5, $A$ and $B$ ). Furthermore, Ad-Brg1/shRRAD HAMSCs showed enhanced proliferation and migration capacity, compared with Ad-Brg1/shScramble HASMCs (Figure 5, $C-G$ ). These results revealed that the negative regulation effects of Brg1 on proliferation and migration in HASMCs were, at least partially, mediated by RRAD.

\section{DISCUSSION}

The major findings of our present study indicated that Brg1 inhibited HASMCs proliferation and migration via 


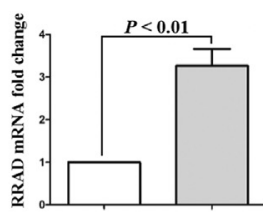

A

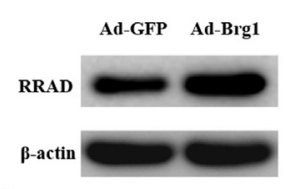

B
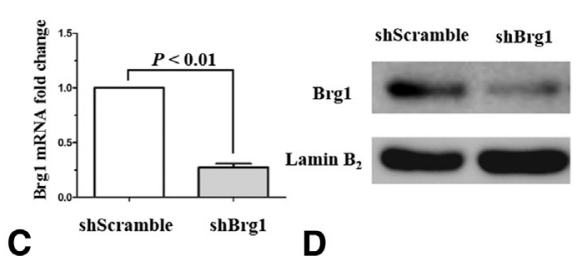

D
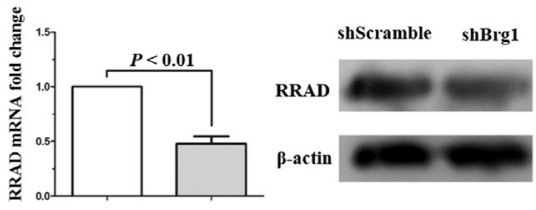

E

hScramble $\quad$ shBrg1

$\mathbf{F}$

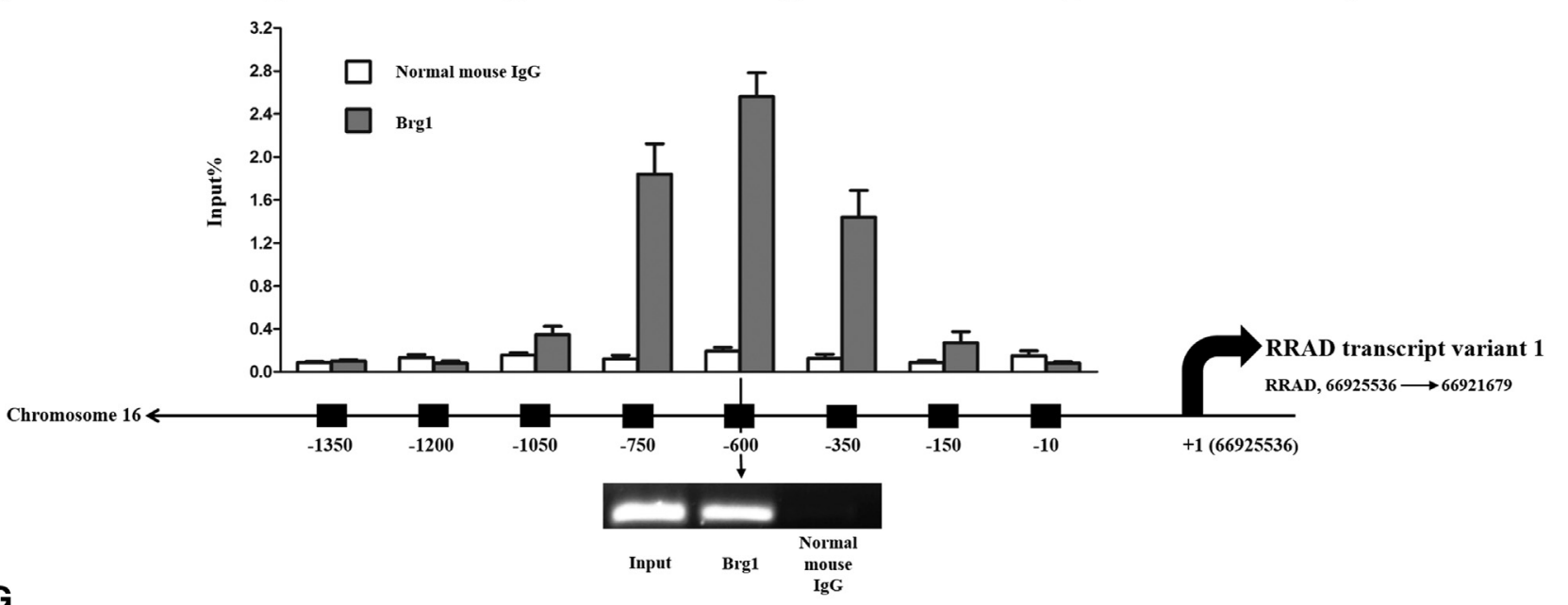

FIGURE 4. Brg1 directly regulated RRAD expression in HASMCs. A and B, The expressions of RRAD were significantly up-regulated at both mRNA and protein levels via overexpression of Brg1 in HASMCs ( $\mathrm{n}=3$ for each cell group). C and D, Successful knockdown of Brg1 in HASMCs via shBrg1. HASMCs transfected with shScramble served as negative control $(n=3$ for each cell group). E and F, RRAD expressions in HASMCs were significantly down-regulated by transfection of $\operatorname{shBrg} 1$ ( $\mathrm{n}=3$ for each cell group). G, Chromatin immunoprecipitation of Brg1 in HASMCs by inducing Brg1 expression with Ad-Brg1, followed by real-time polymerase chain reaction to determine DNA enrichment in the RRAD promoter, relative to input. The schematic representation under the graph is the position of each amplicon relative to the transcription start site ( $\mathrm{n}=3$ for each amplicon position). The bottom corresponds to the $2 \%$ agarose gel of the semi-quantitative polymerase chain reaction of the top. All experiments were carried out in triplicate. $R R A D$, Ras-related associated with diabetes; $m R N A$, messenger ribonucleic acid; $A d-G F P$, a control vector expressing green fluorescent protein; $A d$-Brg1, adenovirus vector coexpressing Brg1 as well as green fluorescent protein; shScramble, negative control short hairpin RNA adenovirus vector; shBrg1, Brg1-specific short hairpin RNA adenovirus vector; Brg1, Brahma-related gene 1; IgG, immunoglobulin.

directly upregulating RRAD, which would be an important mechanism for the pathophysiologic processes of aortic dissection. This conclusion was verified according to our experimental results in the following way. In tissue samples, the expression levels of $\mathrm{Brg} 1$ were higher in $\mathrm{AD}$ patients. Overexpression of Brg 1 in HASMCs considerably inhibited their proliferation and migration.

To elucidate the molecular mechanisms therein, we further screened out RRAD with a PCR array as the candidate proliferation and a migration-related $\mathrm{Brg} 1$ target gene, whose expression levels were higher in dissecting aorta. In vitro experiments further clarified that RRAD was a direct regulation target of $\mathrm{Brg} 1$ during its transcription in HASMCs. Finally, we demonstrated that down-regulating RRAD expression significantly attenuated the repression effects of $\mathrm{Brg} 1$ on proliferation and migration in HASMCs.

As the major cellular components of aortic media, VSMCs are responsible for implementing various biological functions, including vascular remodeling. ${ }^{4,5}$ However, pathologic changes in the biological characteristics of
VSMCs have been reported to have considerable involvement in several aortic diseases. ${ }^{4}$ Aortic remodeling was considered to be a damage-response process, for the aorta to adapt to the change of internal environment, which would have a positive impact on the orderly proliferation and migration of HASMCs. Otherwise, this remodeling would be futile or would have a negative impact. ${ }^{20}$

According to previous studies, the dysfunction of HASMCs in proliferation, via mediation of futile vascular remodeling, played important roles during the pathophysiologic processes of aortic aneurysms and dissection. ${ }^{7,21}$ Briefly, an earlier study revealed that, compared with HASMCs from healthy individuals, HASMCs from patients with aortic aneurysms showed decreased proliferation and migration capacity. ${ }^{22}$ Results of our present study have verified that Brg1 and RRAD mediated the dysfunction of proliferation and migration in HASMCs, which implied the occurrence of a negative aortic remodeling that was beneficial to $\mathrm{AD}$ development.

Extensive study of Brg1 has been conducted in the field of oncology. Decreased expression levels of Brg1 were 


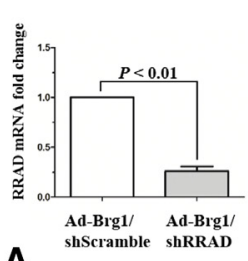

A
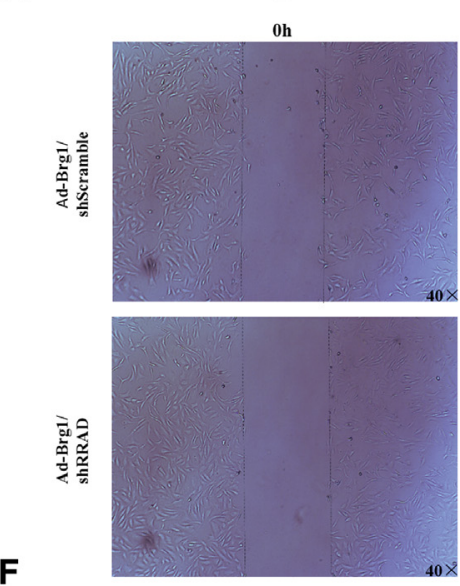

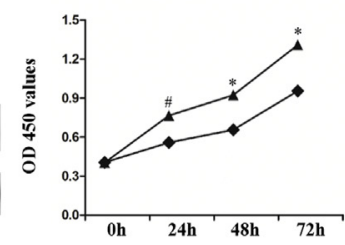

C
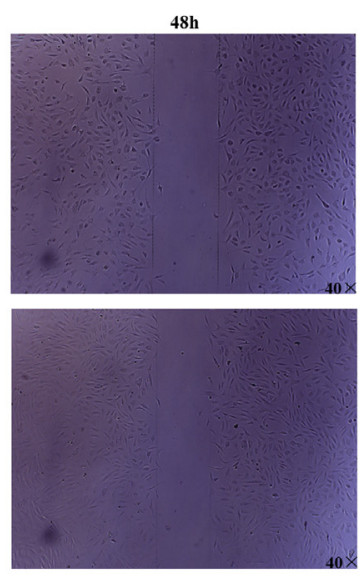

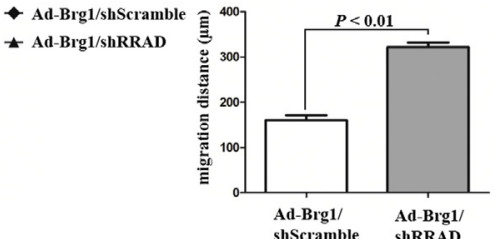

D

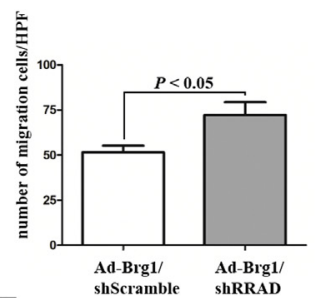

E

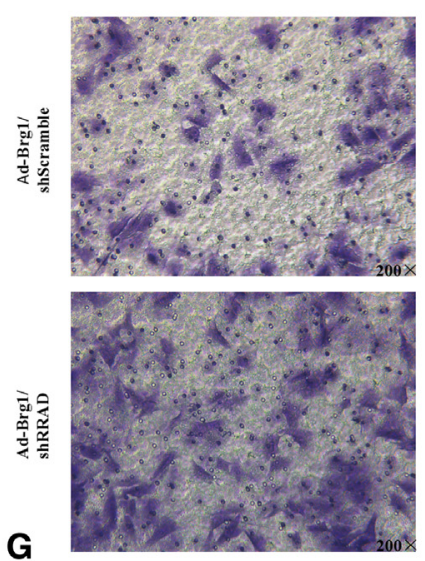

FIGURE 5. The RRAD was a crucial mediator for Brg1 in achieving its suppressive effects on proliferation and migration of HASMCs. A and B, Effective knockdown of RRAD in Ad-Brg1 HASMCs verified by real-time polymerase chain reaction and Western blot ( $\mathrm{n}=3$ for each cell group). Ad-Brg1 HASMCs transfected with shScramble served as negative controls. C, cell counting assay after RRAD knockdown in Ad-Brg1 HASMCs. A higher optical density at $450 \mathrm{~nm}$ was observed in Ad-Brg1/shRRAD HASMCs at 24,48 , and 72 hours after transfection, respectively ( $\mathrm{n}=3$ for each cell group). \#P $<.05$ versus control; $* P<.01$ versus control. D and $\mathrm{F}$, Assessment of migration in HASMCs in 2-dimensional space by wound scratch assay $(\times 40)$. The Ad-Brg1/shRRAD HASMCs showed a significantly higher migration capability. Quantification of migration capacity was measured as migration distance $(\mu \mathrm{m}) ; \mathrm{n}=3$ for each cell group. E and G, Migration chamber assay in Ad-Brg1/shScramble HASMCs and Ad-Brg1/shRRAD HASMCs $(\times 200)$. The Ad-Brg1/shRRAD HASMCs showed a higher migration capacity. Quantification of migration was measured as number of cells per HPF; $\mathrm{n}=3$ for each cell group. All experiments were carried out in triplicate. RRAD, Ras-related associated with diabetes; $m R N A$, messenger ribonucleic acid; Ad-Brgl, adenovirus vector coexpressing Brg1 as well as green fluorescent protein; shScramble, negative control short hairpin RNA adenovirus vector; shRRAD, RRAD-specific short hairpin RNA adenovirus vector; $O D$, optical density; $H P F$, high-power field.

discovered in various clinical tumor tissue specimens, such as non-small cell lung cancer. ${ }^{23}$ According to the study of Hendricks and colleagues, ${ }^{24}$ re-expression of $\mathrm{Brg} 1$ in some specific tumor cell lines would significantly upregulate several tumor suppressors, such as $\mathrm{p} 21$ and $\mathrm{p} 15$, which would thus induce cell-cycle arrest and inhibit tumor-cell proliferation. However, a recent study has reported that Brg1 expression levels were enhanced in the glioma tissues, and that knocking out Brg1 would inhibit proliferation and migration of glioma cells. ${ }^{25}$

This evidence, taken together, implied that Brg1 might play a critical but diversified role in aspects of tumor-cell proliferation and migration, as evaluated by Roy and colleagues. ${ }^{26}$ However, studies concerning the functions of Brg1 in the cardiovascular field were quite limited. Previous studies have reported that Brg1 acted as an indispensable chromatin modulator during the embryonic development of the cardiovascular system, and maintained a low expression level in cardiovascular tissues after adulthood. ${ }^{9,27}$ Other studies ${ }^{13,14,28}$ have reported that Brg1 was involved in the pathophysiologic processes of various cardiovascular diseases. Zhang and colleagues ${ }^{28}$ reported that Brg1 induced VSMC apoptosis in atherosclerosis. Our previous studies showed that Brg1 was involved in aortic diseases via inducing apoptosis of HASMCs. ${ }^{13,14}$ In the present study, we further verified, using human aortic specimens and HASMCs, that Brg1 expression levels increased in $\mathrm{AD}$ patients. Overexpression of $\mathrm{Brg} 1$ significantly inhibited HASMC proliferation and migration.

A small GTPase of the Ras superfamily, RRAD, was recently reported to be a tumor suppressor that would be inactivated via methylation of its promoter. ${ }^{29,30}$ Mo and colleagues $^{29}$ and Suzuki and colleagues ${ }^{30}$ reported that a significantly lower expression, and an elevated promoter methylation level, of RRAD occurred in some human tumor tissues. Emerging data have illuminated the fact that the potential mechanisms within the tumor suppression functions of RRAD may lie in its negative regulation effect on cell migration and invasion, as well as on the processes of glucose uptake and glycolysis. . $^{19,31,32}$ 
In the field of cardiovascular disease, previous studies have verified the protective role of RRAD in preventing cardiac fibrosis and hypertrophy. ${ }^{33,34}$ In addition, Fu and colleagues $^{18}$ have reported that RRAD suppressed migration of VSMCs in both in vitro and in vivo experiments. After RRAD was screened via PCR array as the candidate target gene of Brg1, we revealed for the first time the elevated expression level of RRAD in aortic dissection specimens. We further provided direct evidence that RRAD inhibits proliferation in HASMCs. The effect of RRAD on HASMCs migration in our study was verified using a rescue experiment, which was consistent with the results of $\mathrm{Fu}$ and colleagues. ${ }^{18}$

The direct regulation relationship of Brg1 and RRAD was revealed by our study, via overexpressing and knocking out Brg1 in HASMCs, and utilization of chromatin immunoprecipitation assay. As a new, distinct RRAD regulation pathway, compared with the mechanisms of p53 regulating RRAD in tumor diseases, ${ }^{19,31}$ our results on this particular factor enriched understanding of the regulation network of RRAD.

In summary, our findings suggest that Brg1 plays an important role in the pathophysiologic processes of $\mathrm{AD}$ by inhibiting HASMC proliferation and migration via direct up-regulation of RRAD. Our present study has provided new insights into the roles and relative regulation mechanisms of Brg1 and RRAD in the pathogenesis of AD.

However, our study inevitably had several limitations. We obtained the results using an in vitro cell model, which could not completely simulate the actual situation in $\mathrm{AD}$ pathogenesis. Second, the exact mechanisms accounting for RRAD inhibition of HASMC proliferation and migration were not determined. An earlier study showed that RRAD suppressed VSMC migration by affecting the Rho/ROK (rho-associated kinase) signal pathway, a well established pathway that regulates cell migration. ${ }^{18,35}$ However, whether RRAD participates in other signal pathways to regulate VSMC migration and proliferation needs further study.

Third, we have not proven that Brg1 and RRAD were specifically involved in the pathogenesis of AD. The fact that $\mathrm{Brg} 1$ and RRAD were additionally upregulated in dilated aortic tissue indicated that Brg1 and RRAD might be involved in the pathogenesis of aortic dilation. Fourth, apart from the impact of $\mathrm{Brg} 1$ in regulating apoptosis, proliferation, and migration of HASMCs, its potential role in affecting the biological characteristics of HASMCs and the pathophysiologic processes of aortic dissection, via other mechanisms, should be clarified.

\section{Conflict of Interest Statement}

Authors have nothing to disclose with regard to commercial support.

\section{References}

1. Golledge J, Eagle KA. Acute aortic dissection. Lancet. 2008;372:55-6.

2. LeMaire SA, Russell L. Epidemiology of thoracic aortic dissection. Nat Rev Cardiol. 2011;8:103-13.

3. Rylski B, Beyersdorf F, Blanke P, Boos A, Hoffmann I, Dashkevich A, et al. Supracoronary ascending aortic replacement in patients with acute aortic dissection type A: What happens to the aortic root in the long run? J Thorac Cardiovasc Surg. 2013;146:285-90.

4. Lacolley P, Regnault V, Nicoletti A, Li Z, Michel JB. The vascular smooth muscle cell in arterial pathology: a cell that can take on multiple roles. Cardiovasc Res. 2012;95:194-204.

5. Korshunov VA, Schwartz SM, Berk BC. Vascular remodeling: hemodynamic and biochemical mechanisms underlying Glagoy's phenomenon. Arterioscler Thromb Vasc Biol. 2007; 27:1722-8.

6. Gerthoffer WT. Mechanisms of vascular smooth muscle cell migration. Circ Res 2007; 100:607-21.

7. Milewicz DM, Guo DC, Tran-Fadulu V, Lafont AL, Papke CL, Inamoto S, et al. Genetic basis of thoracic aortic aneurysms and dissections: focus on smooth muscle cell contractile dysfunction. Annu Rev Genomics Hum Genet. 2008;9: 283-302.

8. Ho L, Crabtree GR. Chromatin remodelling during development. Nature. 2010; 463:474-84.

9. Hang CT, Yang J, Han P, Cheng HL, Shang C, Ashley E, et al. Chromatin regulation by $\operatorname{Brg} 1$ underlies heart muscle development and disease. Nature. 2010;466:62-7.

10. Zhang M, Fang H, Zhou J, Herring BP. A novel role of Brgl in the regulation of SRF/MRTFA-dependent smooth muscle-specific gene expression. J Biol Chem. 2007;282:25708-16.

11. Zhou J, Zhang M, Fang H, El-Mounayri O, Rodenberg JM, Imbalzano AN, et al. The SWI/SNF chromatin remodeling complex regulates myocardin-induced smooth muscle-specific gene expression. Arterioscler Thromb Vasc Biol. 2009; 29:921-8.

12. Griffin CT, Curtis CD, Davis RB, Muthukumar V, Magnuson T. The chromatin remodeling enzyme BRG1 modulates vascular Wnt signaling at two levels. Proc Natl Acad Sci. 2011;108:2282-7.

13. Wang S, Zhang X, Yuan Y, Tan M, Zhang L, Xue X, et al. BRG1 expression is increased in thoracic aortic aneurysms and regulates proliferation and apoptosis of vascular smooth muscle cells through the long non-coding RNA HIF1A-AS1 in vitro. Eur J Cardiothorac Surg. 2015;47:439-46.

14. Yuan Y, Wang C, Xu J, Tao J, Xu Z, Huang S. BRG1 overexpression in smooth muscle cells promotes the development of thoracic aortic dissection. BMC Cardiovasc Disord. 2014;14:144.

15. Reynet C, Kahn CR. Rad: a member of the Ras family overexpressed in muscle of type II diabetic humans. Science. 1993;262:1441-4.

16. Zhang XW, Zhang BY, Wang SW, Gong DJ, Han L, Xu ZY, et al. Twist-related protein 1 negatively regulated osteoblastic transdifferentiation of human aortic valve interstitial cells by directly inhibiting runt-related transcription factor 2 . J Thorac Cardiovasc Surg. 2014;148:1700-8.

17. Zhang M, Liu X, Zhang X, Song Z, Han L, He Y, et al. MicroRNA-30b is a multifunctional regulator of aortic valve interstitial cells. J Thorac Cardiovasc Surg. 2014;147:1073-80.

18. Fu M, Zhang J, Tseng YH, Cui T, Zhu X, Xiao Y, et al. Rad GTPase attenuates vascular lesion formation by inhibition of vascular smooth muscle cell migration. Circulation. 2005;111:1071-7.

19. Hsiao BY, Chen CC, Hsieh PC, Chang TK, Yeh YC, Wu YC, et al. Rad is a p53 direct transcriptional target that inhibits cell migration and is frequently silenced in lung carcinoma cells. J Mol Med (Berl). 2011;89:481-92.

20. Yu H, Clarke MC, Figg N, Littlewood TD, Bennett MR. Smooth muscle cell apoptosis promotes vessel remodeling and repair via activation of cell migration, proliferation, and collagen synthesis. Arterioscler Thromb Vasc Biol. 2011;31: 2402-9.

21. Guo DC, Pannu H, Tran-Fadulu V, Papke CL, Yu RK, Avidan N, et al. Mutations in smooth muscle alpha-actin (ACTA2) lead to thoracic aortic aneurysms and dissections. Nat Genet. 2007;39:1488-93.

22. Blunder S, Messner B, Aschacher T, Zeller I, Türkcan A, Wiedemann D, et al. Characteristics of TAV- and BAV-associated thoracic aortic aneurysms - smooth muscle cell biology, expression profiling, and histological analyses. Atherosclerosis. 2012;220:355-61.

23. Reisman DN, Sciarrotta J, Wang W, Funkhouser WK, Weissman BE. Loss of BRG1/BRM in human lung cancer cell lines and primary lung cancers: correlation with poor prognosis. Cancer Res. 2003;63:560-6. 
24. Hendricks KB, Shanahan F, Lees E. Role for BRG1 in cell cycle control and tumor suppression. Mol Cell Biol. 2004;24:362-76.

25. Bai J, Mei PJ, Liu H, Li C, Li W, Wu YP, et al. BRG1 expression is increased in human glioma and controls glioma cell proliferation, migration and invasion in vitro. J Cancer Res Clin Oncol. 2012;138:991-8.

26. Roy N, Malik S, Villanueva KE, Urano A, Lu X, Von Figura G, et al. Brg1 promotes both tumor-suppressive and oncogenic activities at distinct stages of pancreatic cancer formation. Genes Dev. 2015;29:658-71.

27. Griffin CT, Brennan J, Magnuson T. The chromatin-remodeling enzyme BRG1 plays an essential role in primitive erythropoiesis and vascular development. Development. 2008;135:493-500.

28. Zhang QJ, Goddard M, Shanahan C, Shapiro L, Bennett M. Differential gene expression in vascular smooth muscle cells in primary atherosclerosis and in stent stenosis in humans. Arterioscler Thromb Vasc Biol. 2002;22: 2030-6.

29. Mo Y, Midorikawa K, Zhang Z, Zhou X, Ma N, Huang G, et al. Promoter hypermethylation of Ras-related GTPase gene RRAD inactivates a tumor suppressor function in nasopharyngeal carcinoma. Cancer Lett. 2012;323: 147-54.
30. Suzuki M, Shigematsu H, Shames DS, Sunaga N, Takahashi T, Shivapurkar N et al. Methylation and gene silencing of the Ras-related GTPase gene in lung and breast cancers. Ann Surg Oncol. 2007;14:1397-404.

31. Zhang C, Liu J, Wu R, Liang Y, Lin M, Liu J, et al. Tumor suppressor p53 negatively regulates glycolysis stimulated by hypoxia through its target RRAD. Oncotarget. 2014:5:5535-46.

32. Wang Y, Li G, Mao F, Li X, Liu Q, Chen L, et al. Ras-induced epigenetic inactivation of the RRAD (Ras-related associated with diabetes) gene promotes glucose uptake in a human ovarian cancer model. J Biol Chem. 2014;289:14225-38.

33. Zhang J, Chang L, Chen C, Zhang M, Luo Y, Hamblin M, et al. Rad GTPase inhibits cardiac fibrosis through connective tissue growth factor. Cardiovasc Res. 2011;91:90-8

34. Chang L, Zhang J, Tseng YH, Xie CQ, Ilany J, Brüning JC, et al. Rad GTPase deficiency leads to cardiac hypertrophy. Circulation. 2007;116:2976-83.

35. Giancotti FG, Ruoslahti E. Integrin signaling. Science. 1999;285:1028-32.

Key Words: aortic dissection, Brg1, RRAD, human aortic smooth muscle cell, proliferation, migration

\title{
EDITORIAL COMMENTARY
}

\section{Aortic dissection and DNA repair}

\author{
Alden H. Harken, MD
}

From the Department of Surgery, University of California, San Francisco-East Bay, Oakland, Calif. Disclosures: Author has nothing to disclose with regard to commercial support.

Received for publication Aug 21, 2015; accepted for publication Aug 21, 2015; available ahead of print Sept 19, 2015.

Address for reprints: Alden H. Harken, MD, Department of Surgery, University of California, San Francisco-East Bay, 1411 E 31st St, QIC 22134, Oakland, CA 94602 (E-mail: Alden.Harken@ucsfmedctr.org). J Thorac Cardiovasc Surg 2015;150:1301-2

$0022-5223 / \$ 36.00$

Copyright (C) 2015 by The American Association for Thoracic Surgery

http://dx.doi.org/10.1016/j.jtcvs.2015.08.088

The genetic recipe sunk deep into our cells ultimately, somehow, controls both the maturation and behavior of each of our cells. Unfortunately, cells are complex. Imagine taking all the signaling systems and moving parts of a Boeing 747 and packing them into a $20 \mu \mathrm{m}$ space-you've got a cell. Admittedly, there are single-gene-controlled maladies like cystic fibrosis and sickle cell anemia; but, most disease is caused by multiple signaling systems conspiring to accomplish cancer or aortic dissection. Chronic inflammation, with some help from environmental radiation, inflicts as many as 1 million molecular-level lesions per cell per day in each of us-some of which provoke disease. Thus, dysfunctional DNA repair genes like $B r g-1$ and $B R C A-1$ are among the usual suspects when things go awry. Brg-1 expresses an ATP-dependent chromatin remodeling protein that cleans up DNA break damage that otherwise might go rogue.

In the report by Liao and colleagues, ${ }^{1}$ the authors attempt a laudable search for a gene that might eventually serve as a

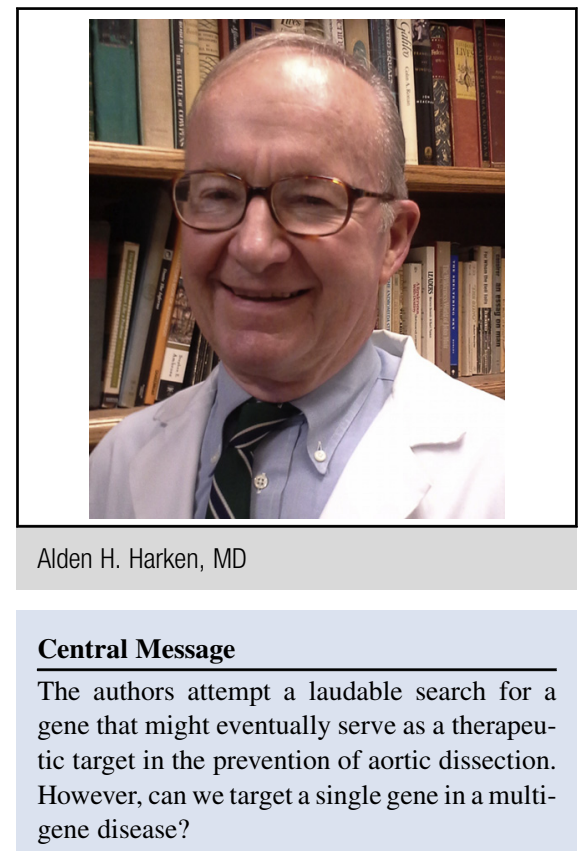

See Article page 1292.

therapeutic target in the prevention of aortic dissection. These authors note that vascular smooth muscle cells (VSMCs) are the most abundant cell type in the aortic media. They therefore postulate that diseased VSMCs are a 


\section{APPENDIX E1.}

The sequences of primers for each gene used in quantitative real-time polymerase chain reaction:

18s rRNA forward: 5'-CGGACACGGACAGGATTGAC; 18s rRNA reverse: 5'-GCATGCCAGAGTCTCGTTCG;

Brg1 forward: 5'-TCATGTTGGCGAGCTATTTCC;

Brg1 reverse: 5'-GGTTCCGAAGTCTCAACGATG;

RRAD forward: 5'-TGAGGTCTCGGTGGATGAGG;

RRAD reverse: 5'-ATGCCGCTGATGTCTCAATG.

\section{CHROMATIN IMMUNOPRECIPITATION}

The chromatin immunoprecipitation (ChIP) assay was performed using the EpiQuik Chromatin Immunoprecipitation Kit (Epigentek, Farmingdale, NY). Twenty-four hours after being transfected with Ad-Brg1, the HASMCs were collected and lysed. The immunoprecipitating antibody was anti-Brg1 antibody (Sc-17796, Santa Cruz Technology). For the negative control, normal mouse IgG (immunoglobulin) in the kit was used. The sequences of primers used were as follows:

RRAD1 forward: 5'-CAGGAAGCCAGAACCAGCAT; RRAD1 reverse: 5'-GCCTGGCTCGGTAAGATTAG;
RRAD2 forward: 5'-TCTTAGGCGTTGCCAAGGA; RRAD2 reverse: 5'-TGATTGTGACAAGTGTGGTGG; RRAD3 forward: 5'-TACCTCACAGCCTGACCTGC; RRAD3 reverse: 5'-TTCGAGTCGTCAAGGTCTCC; RRAD4 forward: 5'-TCCAGGAGCAGAATAACCAGA; RRAD4 reverse: 5'-GTGGAGATGCCTGCTTGAAC; RRAD5 forward: 5'-GTTCAAGCAGGCATCTCCAC; RRAD5 reverse: 5'-TGAGCCTAACCTTCAGTGTGAG; RRAD6 forward: 5'-TCTCTGTCCAGCCTGTAACTAGC; RRAD6 reverse: $5^{\prime}$-TGAGAAGGAGAGAGAGAGCGA; RRAD7forward: 5'-CGCTCTCTCTCTCCTTCTCACA; RRAD7 reverse: 5'-CCTCTTCCTCGGACCTTACA; RRAD8 forward: 5'-ATGTAAGGTCCGAGGAAGAGG; RRAD8 reverse: 5'-ACAGACACGCTCAGGACTAGG.

For RT-PCR, the amplification difference between the negative control and Brg1 varying from 3 to 8 cycles was considered significant. For semi-qPCR, amplifications were performed with 35 cycles in a total volume of $20 \mu \mathrm{L}$, and run on a $2 \%$ agarose gel. 
TABLE E1. Profiles of patients with aortic dilation

\begin{tabular}{lcccccc}
\hline Age $(\mathbf{y})$ and gender & Hypertension & TC $(\mathbf{m m o l} / \mathbf{L})$ & TG $(\mathbf{m m o l} / \mathbf{L})$ & Diabetes & Bicuspid aortic valve & D of AA at its widest $(\mathbf{c m})$ \\
\hline 38, Male & No & 3.52 & 1.43 & No & No & 4.5 \\
56, Female & Yes & 3.87 & 0.96 & No & No & 4.3 \\
53, Male & Yes & 3.12 & 1.57 & Yes & No & 4.7 \\
63, Female & No & 3.97 & 1.12 & No & No & 4.5 \\
\hline
\end{tabular}

$T C$, Serum total cholesterol; $T G$, serum triglyceride; $D$, diameter; $A A$, ascending aorta. 